\title{
Road Impedance Model Study under the Control of Intersection Signal
}

\author{
Yunlin Luo, ${ }^{1}$ Na Wang, ${ }^{1}$ Huaikun Xiang, ${ }^{2}$ and Jinhu Wang ${ }^{3}$ \\ ${ }^{1}$ Aeronautical Automation College, Civil Aviation University of China, Tianjin 300300, China \\ ${ }^{2}$ Automotive and Transportation College, Shenzhen Polytechnic, Shenzhen 518055, China \\ ${ }^{3}$ Tianjin Power Transmission and Distribution Projects Company, Tianjin 300300, China
}

Correspondence should be addressed to Na Wang; huniututu2141@163.com and Huaikun Xiang; xianghuaikun@szpt.edu.cn

Received 20 April 2015; Revised 6 July 2015; Accepted 15 July 2015

Academic Editor: Nazrul Islam

Copyright (C) 2015 Yunlin Luo et al. This is an open access article distributed under the Creative Commons Attribution License, which permits unrestricted use, distribution, and reproduction in any medium, provided the original work is properly cited.

\begin{abstract}
Road traffic impedance model is a difficult and critical point in urban traffic assignment and route guidance. The paper takes a signalized intersection as the research object. On the basis of traditional traffic wave theory including the implementation of traffic wave model and the analysis of vehicles' gathering and dissipating, the road traffic impedance model is researched by determining the basic travel time and waiting delay time. Numerical example results have proved that the proposed model in this paper has received better calculation performance compared to existing model, especially in flat hours. The values of mean absolute percentage error (MAPE) and mean absolute deviation (MAD) are separately reduced by $3.78 \%$ and $2.62 \mathrm{~s}$. It shows that the proposed model has feasibility and availability in road traffic impedance under intersection signal.
\end{abstract}

\section{Introduction}

Road traffic impedance function refers to the relationship between road traveling time and traffic load pressure, which is an important basis of traffic assignment and route guidance. Precise road traffic impedance calculation is a critical technology to make reasonable traffic control decision. It may also effectively avoid wasting travel time and reduce the contamination of environment. With the increasing development of intelligent traffic system (ITS), the signalized intersections become increasingly prevalent in urban roads. Therefore, the study of road traffic impedance grows into a research hotspot. Currently, the road traffic impedance can be divided into two parts: basic travel time and waiting delay time [1].

For the research of basic travel time, the BPR function model proposed by the Federal Highway Administration is the most classical and popular. It is researched by considering the impact of traffic load on the basis of free flow. The research of BPR function has made much progress considering the complex situation of urban traffic flow in China [2]. However, there are still some certain limitations and poor generalization, which still need further study.
For the research of waiting delay time under signalized intersection, the Webster and HCM (1985 and 2000) formulas are typical and widespread. The former is suitable for low traffic load, while the latter is opposite. However, those formulas are not suitable for the intersections in China. The study focuses on improving the classical formulas which have obtained some effects in a number of actual applications [3].

Traffic wave theory is an important branch of traffic flow theory. It describes that the encounter of two traffic flows in the different status with same direction of movement is the transfer process of status which also adheres to the energy conservation law of traffic flow. Thus, the study of road traffic function based on traffic wave theory has attracted wide attention at home and abroad because of its universal adaptability. Traditional traffic wave theory is based on Greenshields linear model [1, 4-9]. Carey and McCartney [1] have analyzed the characteristics of road traffic impedance under the control of intersection signal. Yang [4] has constructed the parking and staring wave model based on Greenberg model, based on analyzing the defects of Greenshields in heavy traffic situation. Yuan [5], Li, and Song [6] and Song et al. [7] have proposed that the road section can be divided into two parts based on queue length: congested and uncongested road 


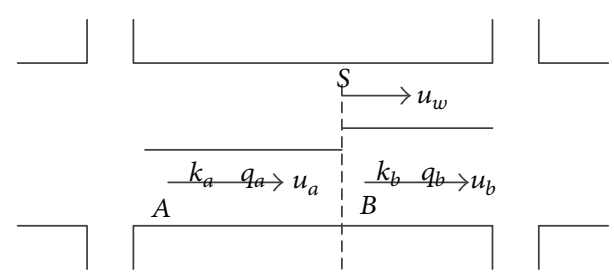

Figure 1: The operation of two different traffic densities.

sections. Then, the road traffic impedance can be calculated separately by each part. It maybe inevitably causes a double error. Wang et al. [8] give the explanations of gathering and dissipating of signalized intersection and analyze different modes under different vehicle tracks. However, it is limited to theoretical research. Sun and Liu [9] have proposed the phase control of intersection signal. The references [10-13] have further analyzed the application of road traffic impedance in intelligent speed estimation, traffic road assignment, and security evaluation of intersection and road network.

In summary, considering the popularity of traditional traffic wave theory, road traffic impedance model under the control of intersection signal is researched based on the Greenshields traffic wave theory with the purpose of obtaining the basic travel time and waiting delay time. The measured data of actual traffic flow and road traffic impedance, in Shenzhen city of Guangdong province of China, is used to conduct calculation and some necessary comparative analysis with the existing model.

The rest of this paper is organized as follows. Section 2 describes the traffic wave theory based on Greenshields model. Section 3 provides the implementation of road impedance model. Section 4 introduces numerical experiment including data collection and results analysis. The conclusion is given in Section 5.

\section{Traffic Wave Theory Based on Greenshields Model}

2.1. Implementation of Traffic Wave Model. As shown in Figure 1, a road section includes two different areas of traffic density $\left(k_{a} \& k_{b}\right)$. The formation of traffic wave is based on the vehicles movement from a density zone ( $A$ zone) to another density zone (zone $B$ ). The cross section to segment the two densities is known as the wave-front $(S)$. The speed of the wave-front is named wave velocity $\left(u_{w}\right)$. From the energy conservation of traffic flow, the number of vehicles which have traveled through the wave-front within time $t$ can be calculated as $N$ :

$$
N=\left(u_{a}-u_{w}\right) k_{a} t=\left(u_{b}-u_{w}\right) k_{b} t
$$

Simplify the equation as follows:

$$
u_{w}=\frac{u_{b} k_{b}-u_{a} k_{a}}{k_{b}-k_{a}} .
$$

On the basis of basic relationships between three parameters of traffic flow, the equation could be simplified as follows:

$$
u_{w}=\frac{q_{b}-q_{a}}{k_{b}-k_{a}} .
$$

Traditional traffic wave model is derived according to the Greenshields model. In order to simplify the equation, the intensity is normalized. In that way, the Greenshields speeddensity model is further calculated as follows:

$$
u=v_{f}\left(1-\frac{k}{k_{j}}\right)=v_{f}(1-\eta) .
$$

By formulas (3) and (4), travel wave model will be obtained as follows:

$$
u_{w}=v_{f}\left[1-\left(\eta_{a}+\eta_{b}\right)\right] .
$$

The parking and starting wave models are researched on the basis of the following analysis.

When the red signal starts, vehicles will stop and gather at the front of parking line with generating a backward propagating wave which is called parking wave. The road section is blocked at the left of wave-front with the standardized density $\eta_{b}=1$. The parking wave model will be obtained as follows:

$$
u_{0}=v_{f}\left[1-\left(\eta_{a}+1\right)\right]=-v_{f} \eta_{a}
$$

When the green signal starts, vehicles will start and dissipate with a forward starting wave. The road section is blocked at the right of wave-front with the standardized density $\eta_{a}=1$. Meanwhile, $u_{b}$ is too small to be negligible.

By formula (4), $\eta_{b}$ will be obtained as follows:

$$
\eta_{b}=1-\left(\frac{u_{b}}{v_{f}}\right) .
$$

Thus, the starting wave model will be obtained as follows:

$$
u_{1}=-v_{f} \eta_{b}=-\left(v_{f}-u_{b}\right) \approx-v_{f} .
$$

In the above formulas, the parameters will be shown as follows:

$u$ : average speed of road section.

$v_{f}$ : travel speed of free flow.

$k_{j}$ : blocking density.

$\eta$ : standardized density.

$\eta_{a}, \eta_{b}:$ standardized density of different adjacent regions.

2.2. Vehicles' Gathering and Dissipating Analysis. As shown in Figure 2, the situation of vehicles' gathering and dissipating under intersection signal will be analyzed. Suppose that there are no vehicles before the red light starting. When the red light starts, vehicles will stop at the parking line and produce parking wave. With the arrival of vehicles at back, the vehicles start queuing. When the green light starts, vehicles will move 


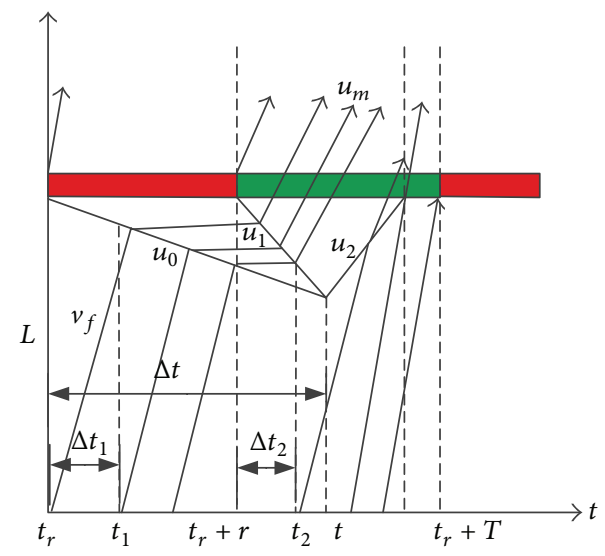

FIGURE 2: Time-space map of signalized intersection.

and produce starting wave. Meanwhile, the length of the queue reaches the maximum when the two traffic waves encounter. Subsequently, the influx of vehicles behind will catch up with the dissipating vehicles and produce forward wave. When forward wave spreads to the stop line, vehicles will arrive at the speed of free flow.

Of course, the absolute value of starting wave velocity must be bigger than parking wave; otherwise, vehicles cannot dissipate forever. At the same time, the parking and starting wave must encounter before the end of the green light; otherwise, vehicles cannot be dissipated within a signal period which will cause queuing more than twice. In this paper, vehicles queue is only considered at most once.

In Figure 2, the parameters will be shown as follows:

$u_{m}$ : speed of dissipating vehicles.

$u_{2}$ : speed of forward wave.

$T$ : time of signal period.

$t_{r}$ : the moment of the red light starting.

$r$ : time of the red light.

$t$ : moment of the two waves encountering.

$t_{1}$ : moment of the vehicle $i$ encountering parking wave.

$t_{2}$ : moment of the vehicle $i$ encountering starting wave.

$L$ : length of road section.

\section{Implementation of Road Traffic Impedance Model}

Road traffic impedance under signal control will be divided into two parts: basic travel time and waiting delay time.

Set $\Delta T$ as road impedance, set $\Delta T_{m}$ as basic travel time, and set $\Delta T_{d}$ as waiting delay time; then, $\Delta T$ will be expressed as follows:

$$
\Delta T=\Delta T_{m}+\Delta T_{d}
$$

3.1. Implementation of Basic Travel Time. Suppose that the speed of the road section is adopted average speed $(u)$ which will solve the difficult problem of measuring the speed $\left(u_{m}\right)$ of dissipating vehicles. Thus, $\Delta T_{m}$ can be easily expressed as follows:

$$
\Delta T_{m}=\frac{L}{u}=\frac{L}{v_{f}(1-\eta)} .
$$

For further calculation, the standardized density $\eta$ can be obtained on the basis of basic relationship of traffic flow parameters and Greenshields linear model which is shown as the following formula:

$$
q=u k=v_{f}\left(1-\frac{k}{k_{j}}\right) k .
$$

So, density $k$ can be obtained through the above solution of a quadratic equation which is shown as follows:

$$
\begin{aligned}
& k_{1}=\frac{1}{2} k_{j}\left(1-\sqrt{1-\frac{4 q}{k_{j} v_{f}}}\right), \\
& k_{2}=\frac{1}{2} k_{j}\left(1+\sqrt{1-\frac{4 q}{k_{j} v_{f}}}\right) .
\end{aligned}
$$

In the current study, $k$ usually appears as absolute value without distinguishing the differences between $k_{1}$ and $k_{2}$. Absolutely, $k_{1}$ is the density of normal driving range and $k_{2}$ is the density of blocking interval in which the speed of vehicles is almost close to 0 . Thus, $k_{1}$ is chosen in this paper considering the actual road section. Then, $\eta$ is shown as follows:

$$
\eta=\frac{k}{k_{j}}=\frac{1}{2}\left(1-\sqrt{1-\frac{4 q l}{v_{f}}}\right) .
$$

In all, basic travel time will get as follows:

$$
\Delta T_{m}=\frac{L}{v_{f}\left[1-(1 / 2)\left(1-\sqrt{1-4 q l / v_{f}}\right)\right]} .
$$

3.2. Implementation of Waiting Delay Time. Suppose that, during observation period, the number of vehicles is $N$ and the probability of vehicles with waiting delay time is $p$. Thus, $\Delta T_{d}$ can be expressed as follows:

$$
\Delta T_{d}=p \sum_{i=1}^{N} \Delta t_{d i}, \quad i=1,2, \ldots, n .
$$

When the vehicle arrives at the road section and encounters the parking wave, it will stop behind the current end of the queue. After waiting a period of time, the vehicle will encounter starting wave and drive away from the intersection. When the vehicle arrive at the range $\left[t_{r}, t\right]$, the vehicle will produce waiting delay time and the probability $(p)$ will be expressed as follows:

$$
p=\frac{\Delta t}{r+g}
$$


It can be further calculated by the following formulas:

$$
\begin{aligned}
u_{0} \Delta t & =u_{1}(\Delta t-r) \\
\Delta t & =\frac{u_{1} r}{u_{1}-u_{0}} \\
p & =\frac{r}{(r+g)(1-\eta)} .
\end{aligned}
$$

At the same time, the waiting delay time of the vehicle $i$ will be expressed as follows:

$$
\Delta t_{d i}=t_{2}-t_{1}=\Delta t_{2}+r-\Delta t_{1} .
$$

It can be further calculated by the following formulas:

$$
\begin{aligned}
\Delta t_{1} & =t_{1}-t_{r}, \\
\Delta t_{2} & =t_{2}-\left(t_{r}+r\right) \\
u_{0} \Delta t_{1} & =u_{1} \Delta t_{2} \\
\Delta t_{d i} & =r-\left(1-\frac{u_{0}}{u_{1}}\right) \Delta t_{1}=r-(1-\eta) \Delta t_{1} .
\end{aligned}
$$

Assuming that the vehicles will queue at most once, no matter when they arrive at the intersection; that is, the maximum waiting delay time of each vehicle is $r$. Thus, waiting delay time of each observation period will be obtained as follows:

$$
\begin{aligned}
\Delta T_{d} & =p \sum_{i=1}^{N} \Delta t_{d i} \\
& =p \frac{1}{r} \int_{0}^{r}\left[r-(1-\eta) \Delta t_{1}\right] d \Delta t_{1} \\
& =p \frac{r(1+\eta)}{2}=\frac{1}{2} \frac{r^{2}(1+\eta)}{(r+g)(1-\eta)} .
\end{aligned}
$$

In summary, road traffic impedance is shown as follows:

$$
\begin{aligned}
\Delta T= & \Delta T_{m}+\Delta T_{d} \\
= & \frac{L}{v_{f}\left[1-(1 / 2)\left(1-\sqrt{1-4 q l / v_{f}}\right)\right]} \\
& +\frac{r^{2}\left[1+(1 / 2)\left(1-\sqrt{1-4 q l / v_{f}}\right)\right]}{2(r+g)\left[1-(1 / 2)\left(1-\sqrt{1-4 q l / v_{f}}\right)\right]} .
\end{aligned}
$$

\section{Numerical Example}

4.1. Data Collection and Calculation Results. Firstly, the road section containing a signalized intersection in Shenzhen city of Guangdong province is selected as data observation object. The purpose of the experiment is to obtain road traffic impedance of each period (one hour) under the signal control. Take the north import of the intersection as an example; the collected data from vehicle inspection device

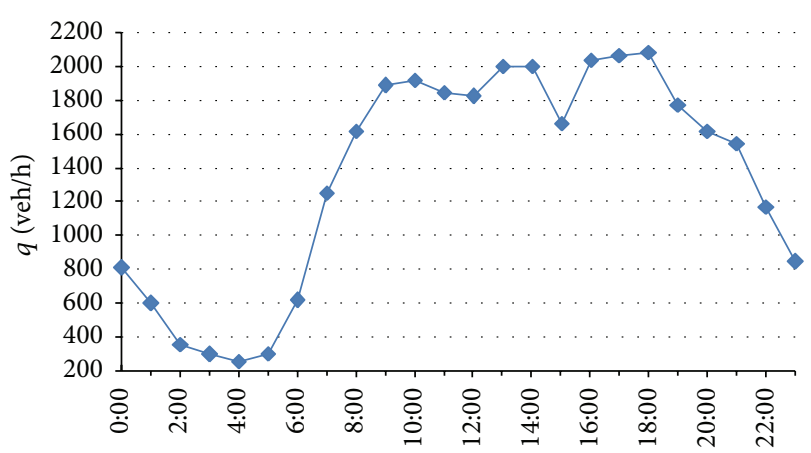

FIGURE 3: Average traffic flow in 24 hours a day.

is only for straight and left turning vehicles, because there exists right turning channelization in the intersection. The green phase of north import is $48 \mathrm{~s}$ and a signal cycle is $108 \mathrm{~s}$. According to the road speed of actual limit situation, the free flow speed is $40 \mathrm{~km} / \mathrm{h}$. The car spacing is $2 \mathrm{~m}$ when the section reaches blocked state. The average length of the car is $5 \mathrm{~m}$. The capacity of north import is $1600 \mathrm{veh} / \mathrm{h}$.

Subsequently, set $500 \mathrm{~m}$ upstream of the intersection as the observation point of road import; in the meanwhile, intersection export is set as the observation point export. The road traffic impedance of every vehicle is obtained through the video capturing license plate of the import and export vehicles.

Thirdly, the data of traffic flow can be obtained from vehicle inspection device on the selected road section.

At last, it is necessary to count the data of road traffic impedance and traffic flow. In order to get more accurate results, five working days are selected to get the average data of one day and the data of road traffic impedance and traffic flow for each period (one hour) are collected. The traffic flow is shown in Figure 3 and actual road traffic impedance is shown in Figure 4 (no separate figure in order to avoid duplication).

In Figure 3, 0:00 represents the time period [23:00-0:00], 1:00 represents the time period [0:00-1:00], 2:00 represents the time period [1:00-2:00], 3:00 represents the time period $[2: 00-3: 00], \ldots, 21: 00$ represents the time period [20:0021:00], 22:00 represents the time period [21:00-22:00], and 23:00 represents the time period [22:00-23:00]. From the curve trend of traffic flow and road traffic impedance, it can be seen that the two curves have the same trend. At the same time, the morning peak hour remains at [9:0010:00], the noon peak hour remains at [13:00-14:00], and the evening peak remains at [17:00-18:00]. The other periods are flat hours.

4.2. Results and Analyses. To evaluate the calculation performance of each model, relative error (RE), mean absolute deviation (MAD), and mean absolute percentage error (MAPE) are calculated as follows and histogram for 24 periods is shown in Figure 5: 


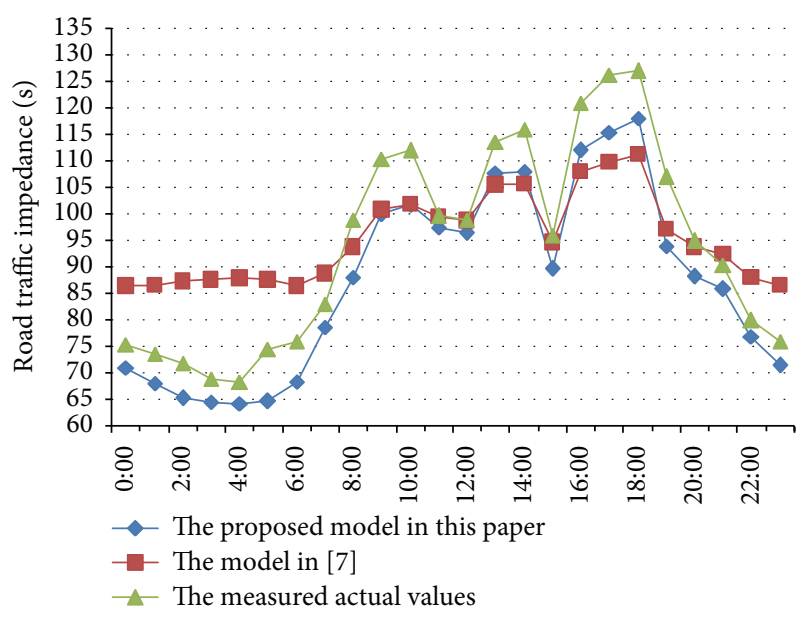

FIGURE 4: The road traffic impedance curve graph.

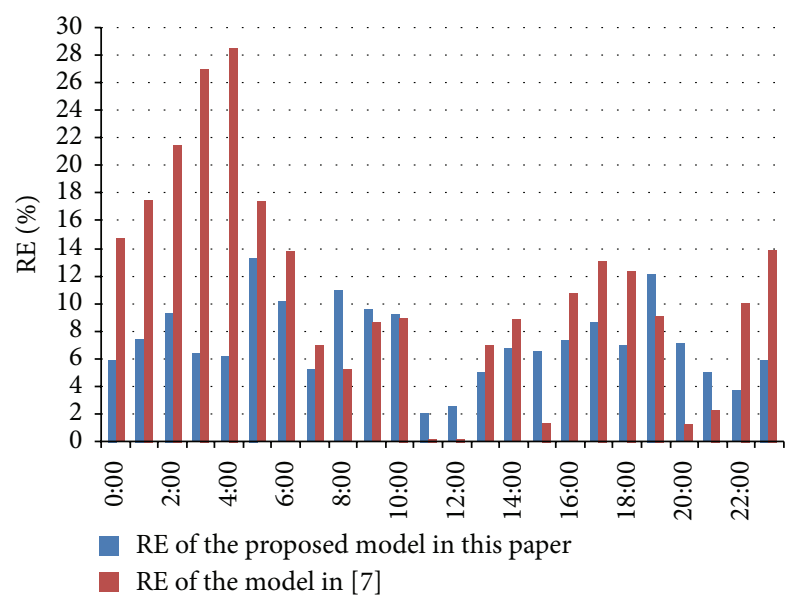

Figure 5: Different road traffic impedance models relative error comparison.

$$
\begin{aligned}
\mathrm{RE} & =\left|\frac{\widehat{Y}_{i}-Y_{i}}{Y_{i}}\right| \times 100 \% \\
\mathrm{MAD} & =\frac{\sum_{i=1}^{n}\left|\widehat{Y}_{i}-Y_{i}\right|}{n} \\
\mathrm{MAPE} & =\frac{\sum_{i=1}^{n}\left|\left(\widehat{Y}_{i}-Y_{i}\right) / Y_{i}\right|}{n} \times 100 \%,
\end{aligned}
$$

in which $\widehat{Y}_{i}$ is the computed value of each period for observation $i, Y_{i}$ is the actual value for observation $i$, and $n$ is the number of computed periods.

Figure 4 shows the curves of the measured actual values and computed values in the two different models (the model in this paper and the model in [7]). The fluctuation of the model in this paper is similar with traffic flow as well. Meanwhile, it is much closer to the measured actual values. So, it is in line with actual road section which demonstrates the feasibility of this model.
TABLE 1: Statistical table of error indexes.

\begin{tabular}{lccc}
\hline Hours & Error indexes & $\begin{array}{c}\text { The model in } \\
\text { this paper }\end{array}$ & $\begin{array}{c}\text { The model } \\
\text { in [7] }\end{array}$ \\
\hline \multirow{2}{*}{ Flat hours } & $\mathrm{MAD} / \mathrm{s}$ & 6.54 & 9.16 \\
& $\mathrm{MAPE} / \%$ & 7.20 & 10.98 \\
\hline \multirow{2}{*}{ Peak hours } & $\mathrm{MAD} / \mathrm{s}$ & 9.03 & 11.98 \\
& $\mathrm{MAPE} / \%$ & 7.67 & 10.04 \\
\hline \multirow{2}{*}{ The whole hours } & $\mathrm{MAD} / \mathrm{s}$ & 6.85 & 9.51 \\
& $\mathrm{MAPE} / \%$ & 7.25 & 10.86 \\
\hline
\end{tabular}

Figure 5 shows the histogram of relative error (RE) values for 24 periods. As shown in Figure 5, the maximum relative error of the model in [7] is more than $28 \%$, while the model in this paper is less than $14 \%$. In order to further analyze the effectiveness of the two different models in the whole hours, flat hours, and peak hours, it is necessary to establish statistical table of error indexes as shown in Table 1.

In Table 1 , on one hand, through horizontal analysis, the MAPE values of the model in this paper are reduced separately by $3.78 \%$ and $2.37 \%$ in flat hours and in peak hours. Meanwhile, the MAD values are separately reduced by $2.62 \mathrm{~s}$ and $2.94 \mathrm{~s}$ in flat hours and in peak hours. In the whole hours, the MAPE and MAD values are reduced separately by $3.61 \%$ and $2.67 \mathrm{~s}$. By horizontal comparison, the model in this paper presents better performance than the model in [7], especially in flat hours.

On the other hand, through longitudinal analysis of the model in this paper, the MAPE value in flat hours is reduced separately by $0.47 \%$ and $0.06 \%$ less than in peak hours and the whole hours, while the MAD value is decreased by $2.49 \mathrm{~s}$ and $0.31 \mathrm{~s}$. By longitudinal comparison, the model in the paper shows excellent feasibility and applicability whether in flat hours, peak hours, or the whole hours.

\section{Conclusion}

Real-time and accurate calculating of road impedance under the control of intersection signal is effective in guiding travel and saving time and energy. This paper proposes road impedance model based on traditional traffic wave theory. Meanwhile, numerical example is employed to conduct the comparison between different models and measured actual situation. The result shows that the model presents better performance. It makes up the deficiency of a double error in the current study. The proposed model is feasible and has a certain engineering value as well.

It should be noticed that waiting delay time under intersection signal is obtained through traditional parking and starting wave theory. Meanwhile, the calculation of basic travel time is done by the average speed of road section which is also based on Greenshields linear relationship. It is well known that Greenshields model is more suitable for the traffic situation of medium density. In this paper, the model has obtained some certain effect at the circumstances of queuing at most once, even though there exist some errors. The model of Greenshields relationship in more severe traffic condition 
still needs to be further researched in urban road section of China.

However, the road traffic impedance under the control of signalized intersection is also affected by other complex factors such as weather conditions, driver or pedestrian behaviors, and emergencies. We still have a long way to go on the research.

\section{Conflict of Interests}

The authors declare that there is no conflict of interests regarding the publication of this paper.

\section{Acknowledgments}

This paper is supported by the Shenzhen city, Guangdong province, basic research project (the project's numbers are JCYJ20120617144302660 and JC201006030851A). The authors appreciate the experimental environment of Shenzhen Polytechnic and thank the students very much because of their hard work to collect data which provide strong support for the completion of this paper.

\section{References}

[1] M. Carey and M. McCartney, "Behaviour of a whole-link travel time model used in dynamic traffic assignment," Transportation Research Part B: Methodological, vol. 36, no. 1, pp. 83-95, 2002.

[2] Transportation Research Board, Highway Capacity Manual, Transportation Research Board, Washington, DC, USA, 2000.

[3] DGJ 08-96-2001, "Intersections of urban road planning and design procedures," A design regulation, Shanghai, China, 2001.

[4] S. H. Yang, Study on the characteristics and control methods of bottleneck forming and diffusing in urban expressway system [Ph.D. thesis], Jilin University, 2006.

[5] Z. H. Yuan, "Study of link travel time functions for dynamic traffic assignment model based on computer simulation," China Journal of Highway and Transport, vol. 15, no. 3, pp. 92-95, 2002.

[6] S. Li and Z. Y. Song, "Macroscopic dynamic link travel time model," Journal of Wuhan University of Technology (Transportation Science \& Engineering), vol. 28, no. 6, pp. 24-29, 2004.

[7] B. W. Song, J. Y. Zhang, Q. Y. Li et al., "Improved dynamic road impedance function based on traffic wave theory," Journal of Chongqing Jiao Tong University (Natural Science), vol. 33, no. 1, pp. 24-29, 2014.

[8] D. H. Wang, H. S. Qi, and Z. H. Li, "Road travel time under signal control," Journal of University (Engineering and Technology Edition), vol. 40, no. 3, pp. 655-660, 2010.

[9] H. Sun and L. Liu, "Study on minimum cycle calculation of intersection signal control by traffic wave theory," Technology \& Economy in Areas of Communications, vol. 61, no. 5, pp. 19-21, 2010.

[10] J. Jin, Z.-J. Qiu, and B. Ran, "Intelligent route-based speed estimation using timing advance," in Proceedings of the IEEE Intelligent Transportation Systems Conference (ITSC '06), pp. 194-197, Toronto, Canada, September 2006.

[11] Q. Zhao, Q.-J. Kong, Y. Xia, and Y. Liu, "Sample size analysis of GPS probe vehicles for urban traffic state estimation," in Proceedings of the 14th IEEE International Intelligent Transportation Systems Conference (ITSC '11), pp. 272-276, October 2011.
[12] X. Y. Liu and X. H. Liu, "Traffic safety assessment method for urban road intersection and its counter measures," China Safety Science Journal, vol. 19, no. 7, pp. 129-134, 2009.

[13] C.-J. Dong, C.-F. Shao, X.-M. Chen, and J. Li, "Road network dynamic splitting based on time-space characteristic of traffic flow," Journal of Jilin University (Engineering and Technology Edition), vol. 40, no. 6, pp. 1528-1532, 2010. 


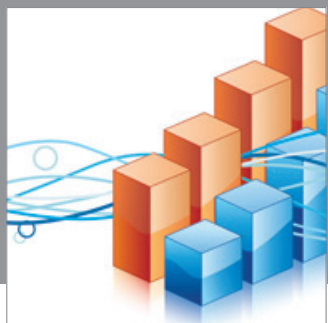

Advances in

Operations Research

mansans

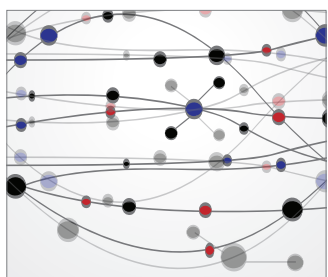

The Scientific World Journal
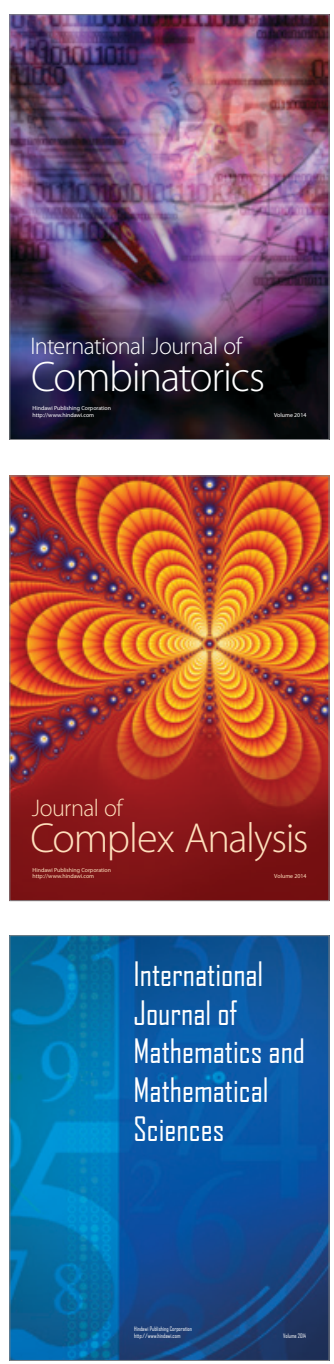
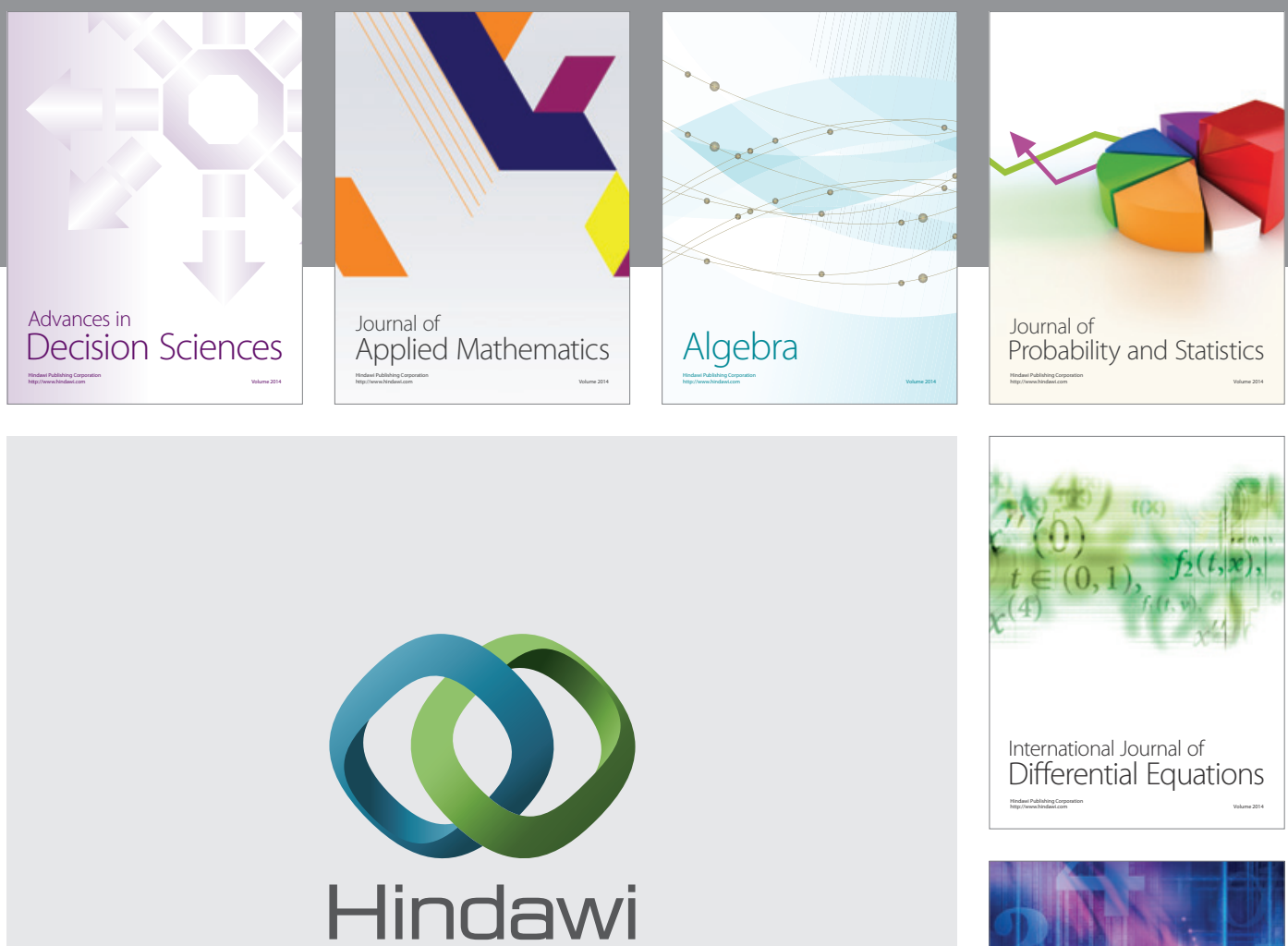

Submit your manuscripts at http://www.hindawi.com
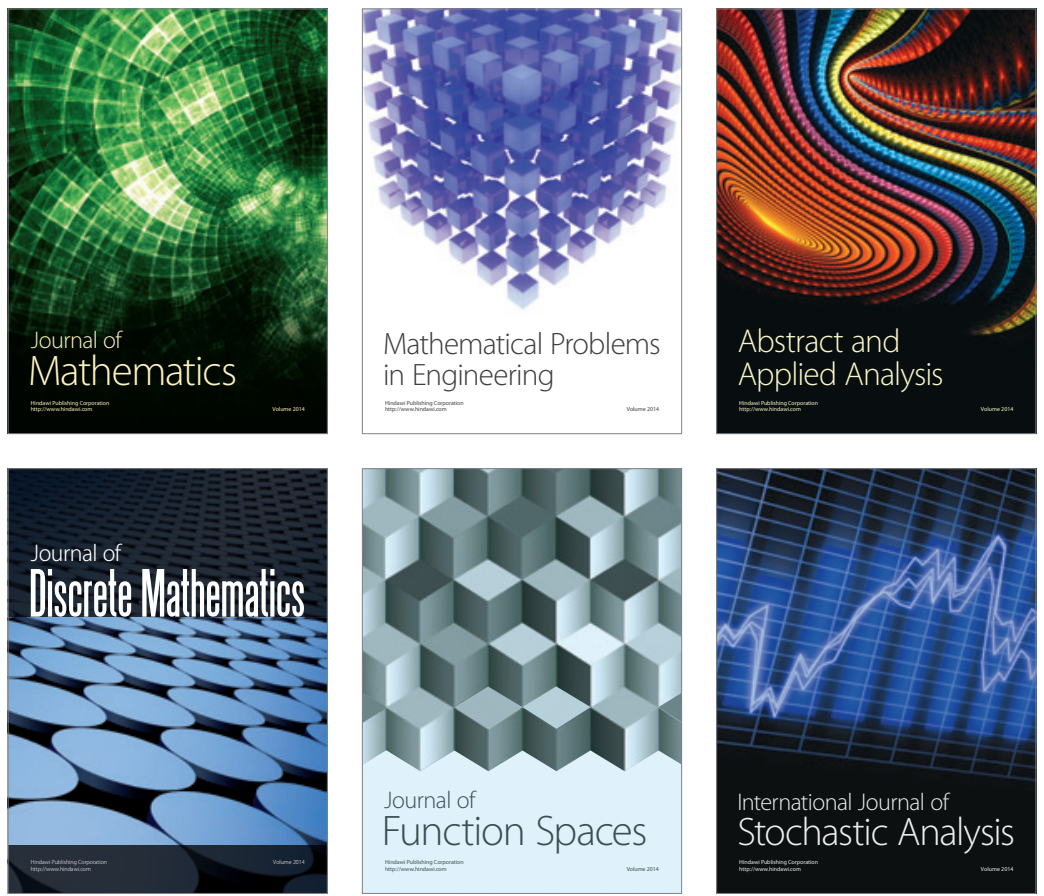

Journal of

Function Spaces

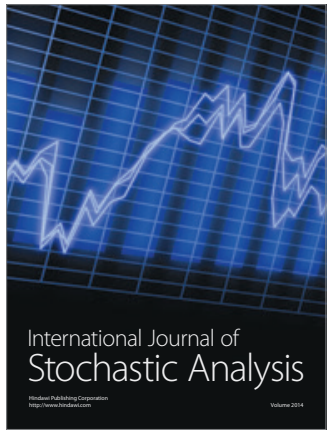

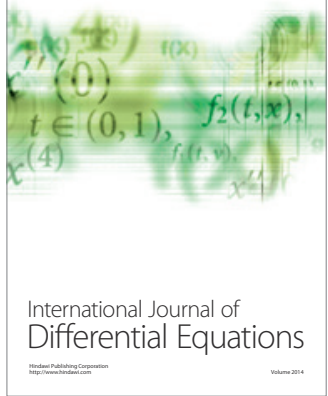
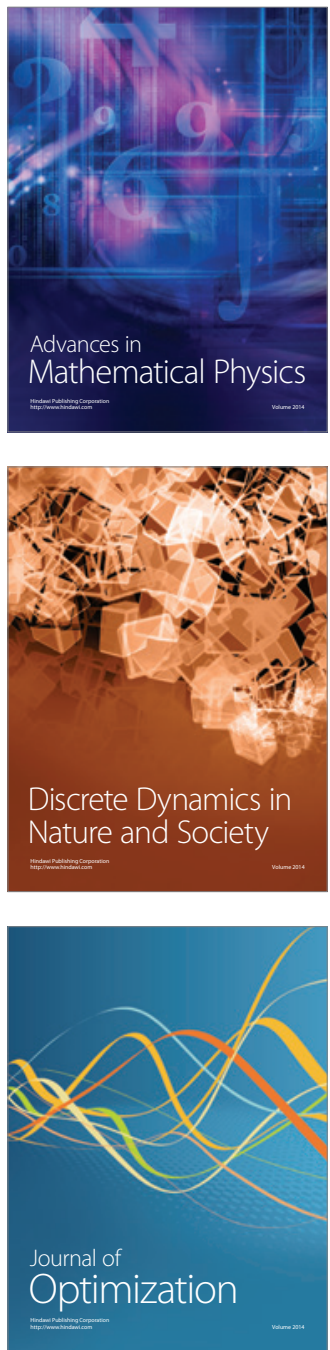